\title{
ADENOSINE A1 RECEPTOR AGONIST PROTECTS AGAINST HIPPOCAMPAL NEURONAL INJURY AFTER LITHIUM CHLORIDE-PILOCARPINE- INDUCED EPILEPSY
}

\author{
Qinua Xiao, hai Tang, Lingwen Kong, Huiming Ji, Ya'nan liu and Guiyun CuI \\ Epilepsy Center, The Affiliated Hospital of Xuzhou Medical University, Xuzhou, China
}

\begin{abstract}
Background: Adenosine A1 receptor (AA1R) is widely present in the central nervous system, exerting brain protective antiepileptic effects, mainly by binding corresponding $G$ proteins. We evaluated the neuroprotective effects of AA1R on hippocampal neuronal injury after lithium chloride-pilocarpine-induced epilepsy in rats. Materials and Methods: A total of 60 male SD rats were randomly divided into four groups ( $n=15$ /group): normal control, epilepsy, epilepsy + AA1R antagonist (DPCPX), and epilepsy + AA1R agonist (2-CAdo). An epilepsy model was established through kindling by lithium chloride-pilocarpine. The four groups were observed on days 1, 14, and 30. Pathological and morphological changes of hippocampal neurons were observed by HE staining; apoptosis was detected by TUNEL assay. Caspase-3 and GABA receptor expressions were detected by Western blot. Results: In the hippocampal CA3 area of the epilepsy group, the cellular structure was not neatly arranged, and some neurons were swelling, thick, and incomplete. Compared with the epilepsy group at the same time point, cells in the epilepsy + DPCPX group had an increased distortion, disorganization, edema, cytoplasmic vacuoles, and degeneration. In the epilepsy + 2-CAdo group, cell arrangement was regular and orderly, and structural damages were lessened. Compared with the normal control group at the same time point, the epilepsy group underwent evident neuronal apoptosis, with a significantly higher apoptotic index $(\mathrm{Al})(p<0.05)$. Compared with the epilepsy group, the neuronal apoptosis of the epilepsy + DPCPX group was boosted, and the Al significantly increased $(p<0.05)$. The neuronal apoptosis of the epilepsy +2 -CAdo group was inhibited, and the Al significantly decreased $(p<0.05)$. Compared with the epilepsy group, the caspase-3 expression levels of the epilepsy + DPCPX group on days 14 and 30 were significantly upregulated $(p<0.05)$, but those of the epilepsy +2 -CAdo group were significantly downregulated $(p<0.05)$. Conclusions: AA1R abated cell edema and reduced apoptosis, exerting neuroprotective effects on hippocampal neuronal injury after lithium chloride-pilocarpine-induced epilepsy. (REV INVEST CLIN. 2019;71:116-23)
\end{abstract}

Key words: Epilepsy. Hippocampus. Neuroprotective agents. Receptor. Adenosine A1.

Corresponding author:

*Guiyun Cui

Epilepsy Center

The Affiliated Hospital of Xuzhou Medical University

Xuzhou 221002, P. R. China

E-mail: cuiguiyunahxmu@yeah.net

Received for publication: 29-06-2018

Approved for publication: 18-09-2018

DOI: $10.24875 / R I C .18002650$ 


\section{INTRODUCTION}

Epilepsy is a central nervous system (CNS) dysfunction syndrome caused by synchronized overdischarge of brain neurons due to a variety of reasons, with recurrent, stereotyped, paroxysmal, and transient characteristics ${ }^{1,2}$. At present, there are two hypotheses to explain refractory epilepsy resistance, the multidrug-resistant transporter hypothesis in the brain, and the sensitivity reduction of drug targets hypothesis ${ }^{3,4}$. Therefore, the adenosine system, as a regulator of endogenous antiepileptic effects in the brain, has attracted widespread attention. Adenosine A1 receptor (AA1R) in the adenosine receptor family is widely present in the CNS, especially with high expressions in the cerebral cortex and hippocampus ${ }^{5}$, exerting brain protective and antiepileptic effects, mainly by binding corresponding $G$ proteins. If exogenous drugs are used to activate AA1R or to increase the concentration of endogenous adenosine, AA1R can protect the brain and combat epilepsy, but the underlying mechanisms are still largely unknown ${ }^{6-8}$. Epileptic seizures are closely related to neuronal apoptosis. After seizures, CNS neurons are selectively damaged, leading to plastic changes in functional structures such as glial proliferation, mossy fiber budding and synaptic structural remodeling. In addition, these changes may aggravate seizures, eventually causing uncontrollable and recurrent epilepsy ${ }^{9}$. GABA receptor is a crucial inhibitory neurotransmitter of the CNS, the level of abnormality of which has been associated with many neurological and psychiatric diseases, also with sedative and anxiolytic effects ${ }^{10}$. Stubbs et al. reported that increasing the expression of GABA receptor, a target of antiepileptic drugs, could control epileptic seizures, but its relationship with $A A 1 R$ remained elusive ${ }^{11}$. AA1R can protect against neuronal injury of ischemic cerebrovascular diseases through various molecular biological mechanisms. However, whether AA1R has neuroprotective effects on seizure-induced hippocampal neuronal injury is still unclear. Thereby motivated, we herein assessed the neuroprotective effects of AA1R on hippocampal neuronal injury after lithium chloride-pilocarpine-induced epilepsy.

\section{MATERIALS AND METHODS}

\section{Experimental animals}

Male SD rats (Sprague Dawley rats grade) weighing 200-250 g were purchased from Beijing, Experimental
Animal Breeding Center (China). The animals were kept under the following standard experimental conditions: a light/dark cycle of $12 \mathrm{~h} / 12 \mathrm{~h}$; without noise; feeding in individual cages; temperature of $18-25^{\circ} \mathrm{C}$; and relative humidity of $40-70 \%$. The experimental process was reviewed and approved by the ethics committee of our hospital. The rats were given adequate food and water before and after the experiment, and their pain was minimized throughout the experiment.

\section{Animal grouping}

A total of 60 male rats were randomly divided into four groups of 15 rats each: a control group, an epilepsy group, an epilepsy + AA1R antagonist (DPCPX) group, and an epilepsy + AA1R agonist (2-CAdo) group. The four groups were observed at three time points, on days 1,14 , and 30 , with five rats for each time point.

The epilepsy group was given $125 \mathrm{mg} / \mathrm{kg}$ lithium chloride by intraperitoneal injection and $20 \mathrm{mg} / \mathrm{kg}$ pilocarpine $20 \mathrm{~h}$ later. The Racine staging was employed for categorizing epileptic seizures: Stage 0: without behavioral changes; Stage 1: mouth and facial movement; Stage 2: head nodding; Stage 3: forelimb clonus; Stage 4: rearing with forelimb clonus; and Stage 5: rearing and falling with forelimb clonus ${ }^{12}$. The epilepsy model was considered successful if Stage 4-5 seizures occurred. The rats were given atropine ( 1 $\mathrm{mg} / \mathrm{kg}$ ) by intraperitoneal injection 30 min after continuous seizures. 30 min later, they were further injected intraperitoneally with diazepam (10 mg/kg) and $10 \%$ chloral hydrate $(5 \mathrm{mg} / \mathrm{kg}$ ) during a convulsion in case of imminent danger. After the seizures were controlled, the rats were subjected to heat preservation and fed in individual cages after waking up, with free access to food and water. For the epilepsy + DPCPX group, DPCPX was injected into the tail vein $1 \mathrm{~h}$ before intraperitoneal injection of lithium chloride-pilocarpine and $1 \mathrm{~h}$ after convulsions at a dose of $0.3 \mathrm{mg} / \mathrm{kg}$. As to the epilepsy + 2-CAdo group, 2-CAdo was injected into the tail vein $1 \mathrm{~h}$ before intraperitoneal injection of lithium chloride-pilocarpine and $1 \mathrm{~h}$ after convulsions at a dose of $0.6 \mathrm{mg} / \mathrm{kg}$.

\section{Histopathological procedures}

All rats were sacrificed under anesthesia to obtain the brain tissue specimen which was fixed with $10 \%$ 
formalin for $24 \mathrm{~h}$ to prepare paraffin sections. The sections were deparaffinized with xylene and rinsed with water after each concentration of ethanol: xylene I, II, 5 min each; absolute ethanol twice, 2 min each; 95\% ethanol, $1 \mathrm{~min} ; 90 \%$ ethanol, $1 \mathrm{~min} ; 80 \%$ ethanol, $1 \mathrm{~min}$; and $70 \%$ ethanol, $1 \mathrm{~min}$. Subsequently, the sections were rinsed twice with distilled water, stained by hematoxylin for $5 \mathrm{~min}$, rinsed with tap water, and differentiated in hydrochloric acid-ethanol for $10 \mathrm{~s}$, followed by immersion in tap water for $10 \mathrm{~min}$, in 75\% ethanol for $2 \mathrm{~min}$, and in $85 \%$ ethanol for $2 \mathrm{~min}$. Then, the sections were subjected to eosin staining for 2 min, conventional dehydration and transparency: $95 \%$ ethanol, $5 \mathrm{~min}$; absolute ethanol, $5 \mathrm{~min}$; and xylene I, II, 5 min each. Finally, the sections were mounted with neutral resin and observed under a light microscope.

\section{Detection of neuron apoptosis by TUNEL assay}

TUNEL assay kit (Thermo Fisher Scientific, USA) was used for the detection of apoptosis. For the hippocampus tissue section of each sample, five visual fields of 400-fold light microscope were randomly selected. After TUNEL staining, the nuclei of positive apoptotic neurons were brown or tan. The total number of cells and the number of TUNEL-positive cells were calculated, respectively, and averaged. The apoptotic index (AI) is the number of TUNEL-positive cells divided by the total number of cells per visual field.

\section{Western blot}

The hippocampus was separated from brain tissue, added RIPA lysis buffer ( $150 \mu \mathrm{l} / 20 \mathrm{mg}$ ), homogenized, and centrifuged at $12,000 \mathrm{rpm}$ for $5 \mathrm{~min}$. Then, the supernatant was collected into an EP tube, and the protein concentration was measured with a BCA protein quantification kit (Beyotime Institute of Biotechnology, China). Each sample ( $50 \mu \mathrm{g}$ ) was subjected to sodium dodecyl-sulfate polyacrylamide gel electrophoresis, and the product was electronically transferred onto a nitrocellulose membrane. Subsequently, the membrane was blocked with $5 \%$ skimmed milk at room temperature for $1 \mathrm{~h}$, incubated overnight with primary antibodies at $4^{\circ} \mathrm{C}$, washed, and incubated with the corresponding secondary antibodies at $37^{\circ} \mathrm{C}$ for $1 \mathrm{~h}$. After developing and scanning, relative protein expressions were detected by Quantity One software after correction with internal reference GAPDH.

\section{Statistical analysis}

All data were analyzed by SPSS 22.0 and expressed as mean \pm standard deviation. The data were subjected to one-way analysis of variance, and intergroup comparisons were performed by the $t$-test. $p<0.05$ was considered statistically significant.

\section{RESULTS}

\section{Effects of AA1R on pathological and morphological changes of neurons in the hippocampal CA3 area after injury}

On days 1,14 , and 30 , neurons in the hippocampal CA3 area of the normal control group were neatly arranged, and the nuclei were round or oval, with intact structures (Fig. 1a).

In the epilepsy group, the cellular structure was not neatly arranged, and some neurons were swelling, thick, and incomplete. With prolonged time, the neurons became disorderly arranged, with unclear boundary, nuclear condensation, shrinkage, and cytoplasmic vacuoles. Meanwhile, the neurons lost their normal structure, undergoing evident degeneration (Fig. 1b).

Compared with the epilepsy group at the same time point, the epilepsy + DPCPX group had aggravated distortion, disorganization, edema, cytoplasmic vacuoles, degeneration, and other structural damages (Fig. 1c). In the epilepsy + 2-CAdo group, the cell arrangement was basically regular and orderly, and structural damages, such as edema, cytoplasmic vacuoles, and degeneration, were lessened (Fig. 1d).

\section{Antiapoptotic effects of AA1R on neurons in the hippocampal CA3 area after injury}

On days 1,14 , and 30 , TUNEL assay of hippocampal CA3 neurons showed that Al values of the normal control group on days 1,14 , and 30 were similar ( $p>0.05$ ) (Fig. $2 \mathrm{a}$ and Table 1). Compared with the normal control group at the same time point, the epilepsy group underwent evident neuronal apoptosis, with a significantly higher Al $(p<0.05)$ (Fig. $2 \mathrm{~b}$ and Table 1 ). 
Figure 1 . HE staining results of neurons in the hippocampal CA3 area on days 1,14 , and 30 . (a) Normal control group, (b) epilepsy group, (c) epilepsy + DPCPX group, (d) epilepsy + 2-CAdo group.

$1 \mathrm{~d}$

a

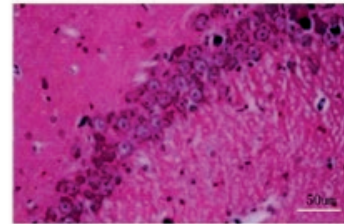

$\mathrm{b}$

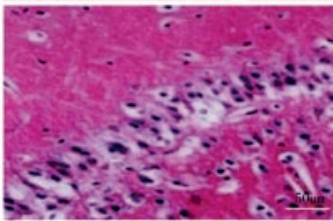

c

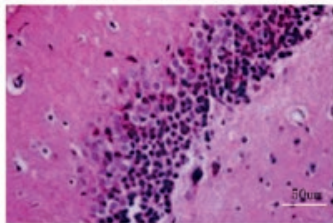

d

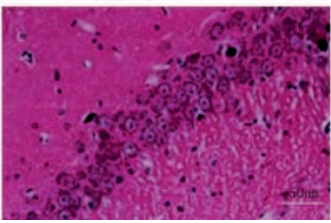

$14 d$
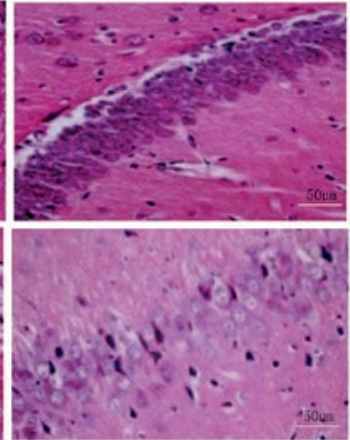
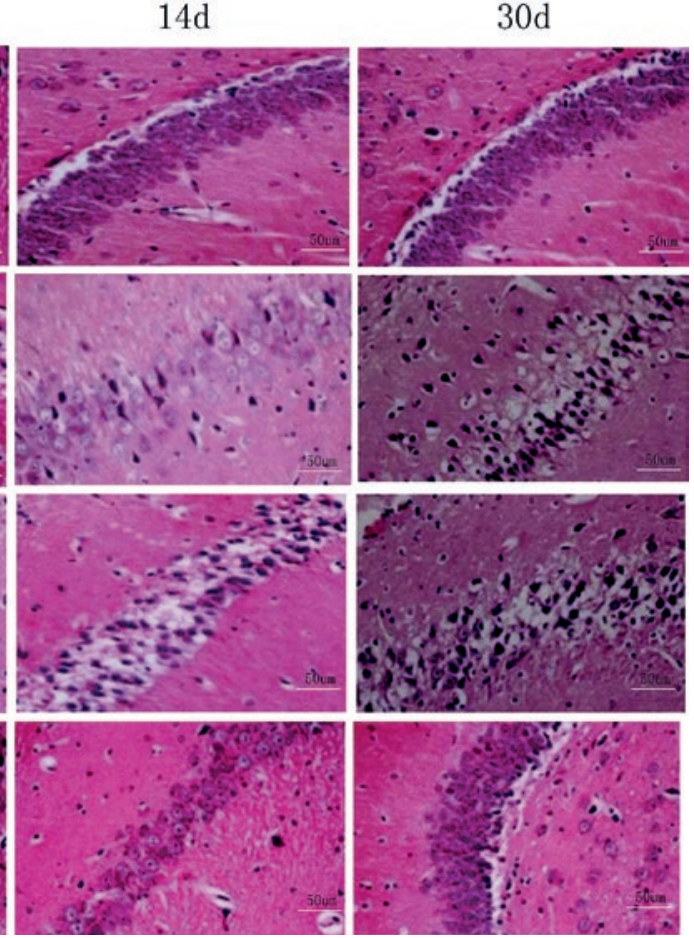

Compared with the epilepsy group, the neuronal apoptosis of the epilepsy + DPCPX group was increased, and Al was also significantly raised $(p<0.05$ ) (Fig. $2 c$ and Table 1). The neuronal apoptosis of the epilepsy + 2-CAdo group was inhibited, and Al was significantly reduced $(p<0.05)$ (Fig. $2 d)$.

\section{Effects of AA1R on caspase-3 protein expression after injury}

Western blot showed that the expressions of caspase- 3 in the normal control group on days 1,14 , and 30 were similar $(p>0.05)$. Compared with the normal control group, the expression levels of the epilepsy group on days 1 and 14 were similar, but levels in day 30 were significantly upregulated $(p<$ 0.05). Compared with the epilepsy group, the expression level of the epilepsy + DPCPX group was similar in day 1 , whereas those on days 14 and 30 were significantly upregulated $(p<0.05)$. Compared with the epilepsy group, the level of the epilepsy + 2-CAdo group was not significantly different in day 1 , but those on days 14 and 30 were significantly downregulated ( $p<0.05$ ) (Fig. 3 ).

\section{Effects of AA1R on GABA receptor expression after injury}

The expression levels of GABA receptor in the normal group were similar on days 1,14 , and 30 ( $p>0.05$ ). With extended time, the level of the epilepsy group decreased. At the same time point, the level was significantly downregulated compared with that of the normal control group ( $p<0.05)$. Compared with the epilepsy group at the same time point, the GABA expression level of the epilepsy + DPCPX group was significantly downregulated ( $p<0.05$ ), whereas that of the epilepsy + 2-CAdo group was significantly upregulated ( $p<0.05$ ) (Fig. 4 ).

\section{DISCUSSION}

The pathogenesis of epilepsy is complex and diverse, which is related to many factors. To date, a variety of epilepsy models have been established, such as those induced by chemical agents and electrical stimulation. The epilepsy model induced by intraperitoneal injection of lithium chloride-pilocarpine has unique 
Figure 2. TUNEL staining results of neurons in the hippocampal CA3 area on days 1, 14, and 30. (a) Normal control group, (b) epilepsy group, (c) epilepsy + DPCPX group, (d) epilepsy + 2-CAdo group. *Comparison between normal control and epilepsy groups, $p<0.05$; \#comparison between epilepsy and epilepsy + DPCPX groups, $p<0.05 ; \Delta$ comparison between epilepsy and epilepsy +2 -CAdo groups, $p<0.05$.

$1 \mathrm{~d}$

a

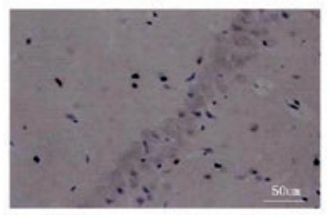

$\mathrm{b}$

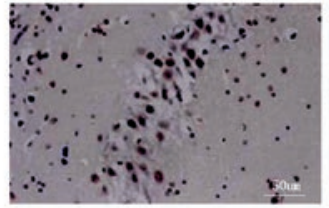

$14 \mathrm{~d}$
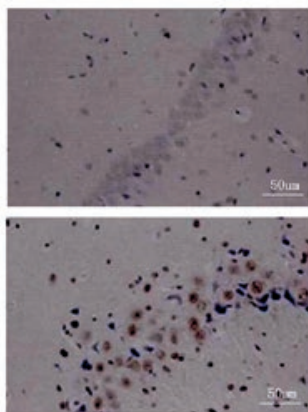

$30 \mathrm{~d}$
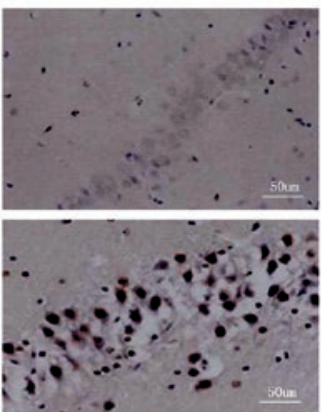

c
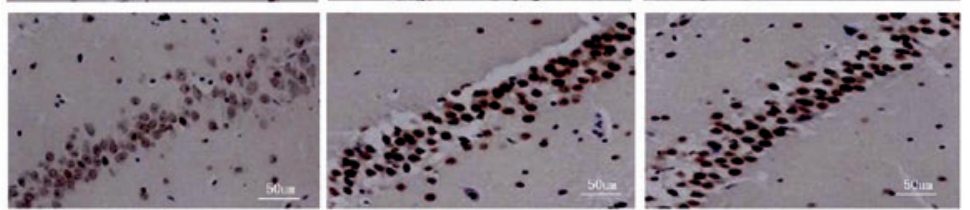

d
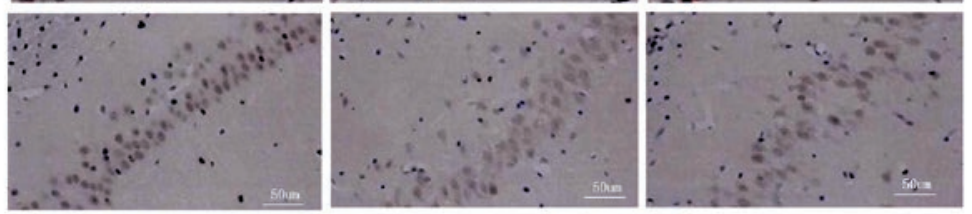

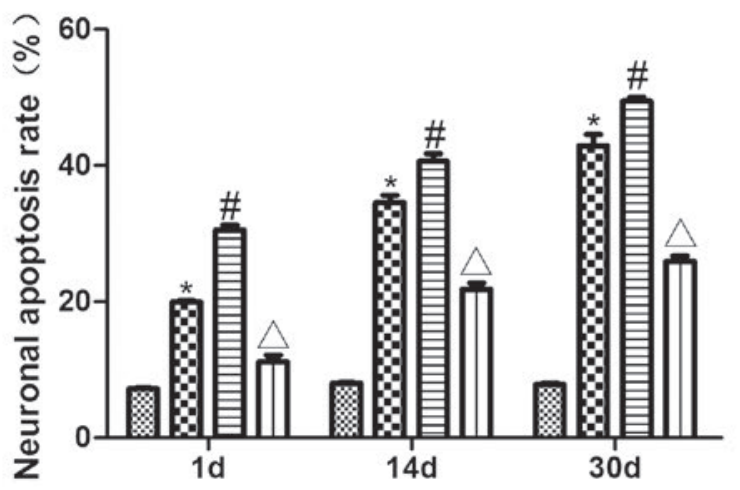

Normal control

$\mathbf{\infty}$ Epilepsy

E Epilepsy + DPCPX

而 epilepsy + 2-CAdo

pathophysiological processes ${ }^{13}$. The changes in EEG of rats during seizures are similar to those in humans. The epilepsy model enters the chronic phase 15 days after acute seizures when rats undergo spontaneous seizures in a repeated and intermittent way ${ }^{14-16}$. In this study, the success rate and mortality rate of the lithium chloride-pilocarpine induction-induced epilepsy model were $82.6 \%$ and $10.0 \%$, respectively. In addition, this model has basic pathological changes similar to those of human temporal lobe epilepsy ${ }^{17}$. It is the most classical chemical-induced animal model for the study of temporal lobe epilepsy, which can specifically damage the hippocampus, causing hippocampal neuronal injury and apoptosis.

Epilepsy is closely related to neuronal apoptosis. Epileptic seizures can induce neuronal apoptosis, which can, in turn, also aggravate the seizures. This study confirmed that neurons were neatly arranged with a clear contour, and the nuclei were round or oval, with intact structures. HE staining of the specimen from the epilepsy group showed that 1 day after seizures, the structure of hippocampal neurons was not neatly arranged, and some cells were swelling, thick, and 
Table 1. Al values of neurons in the hippocampal CA3 area on days 1,14 , and 30 (\%).

\begin{tabular}{|c|c|c|c|}
\hline \multirow[t]{2}{*}{ Group } & \multicolumn{3}{|l|}{$\mathrm{Al}(\%)$} \\
\hline & Day 1 & Day 14 & Day 30 \\
\hline Normal control & $7.15 \pm 0.17$ & $8.07 \pm 0.21$ & $7.67 \pm 0.19$ \\
\hline Epilepsy & $19.87 \pm 2.18^{a}$ & $33.41 \pm 1.98^{a}$ & $41.23 \pm 1.88^{a}$ \\
\hline Epilepsy + DPCPX & $29.86 \pm 2.09^{b}$ & $40.78 \pm 1.96^{b}$ & $48.81 \pm 1.69^{b}$ \\
\hline Epilepsy + 2-CAdo & $10.97 \pm 1.18^{c}$ & $22.67 \pm 1.76^{c}$ & $25.49 \pm 1.79^{c}$ \\
\hline
\end{tabular}

${ }^{a} p<0.05$, comparison between epilepsy and normal control groups at each time point

${ }^{b} p<0.05$, comparison between epilepsy + DPCPX group and epilepsy group at each time point

${ }^{c} p<0.05$, comparison between epilepsy +2 -CAdo group and epilepsy group at each time point

Al: apoptotic index

incomplete. With extended time, the neurons were arranged disorderly on days 14 and 30 , with ambiguous contour, unclear boundary, nuclear condensation, shrinkage, cytoplasmic vacuoles, and degeneration. Meanwhile, the gap between cells became wider. Thus, epilepsy indeed caused hippocampal neuronal damage, being consistent with the results of André et al. ${ }^{18}$ and Hanaya et al. ${ }^{19}$

In this study, TUNEL assay showed that, compared with the normal control group at the same time point, there was evident neuronal apoptosis in the epilepsy group, which was gradually enhanced on days 1,14 , and 30. Compared with the normal control group, the Al of neurons in the epilepsy group was significantly higher than that of the normal control group ( $p<$ 0.05). Thus, seizures induced hippocampal neuronal apoptosis, being in accordance with the study of Patel et al. ${ }^{20}$ However, the mechanism of neuronal apoptosis after seizures has not yet been fully elucidated, which may be related to the following aspects: (1) seizures lead to neurotransmitter changes in the brain, release considerable excitatory amino acid Glu, which can open channels for $\mathrm{Na}^{+}, \mathrm{Cl}^{-}$, and water molecules, causing neuronal swelling followed by neuronal apoptosis; (2) Glu also acts on postsynaptic NMDA and non-NMDA receptors, so $\mathrm{Ca}^{2+}$ enters cells, which can increase intracellular $\mathrm{Ca}^{2+}$ concentration, activate voltage-dependent calcium channels, and ultimately cause neuronal degeneration; (3) Glu increase and calcium ion inflow open the permeable pores of mitochondrial membrane, leading to mitochondrial damage and then neuronal apoptosis. Moreover, opening of the pores aggravates neuronal damage by releasing cytochrome $\mathrm{C}$; and (4) seizures affect the regulation of neuronal apoptosis-related gene expressions and signaling pathways. Using an animal model of lithium chloride-pilocarpine-induced epilepsy, Tan et al. confirmed that $P 53$ gene and caspase- 3 were associated with neuronal apoptosis ${ }^{21}$. Another study also verified that neuronal damage was related to the MAPK signaling pathway after seizures. In short, neuronal damage and apoptosis after seizures are associated with the toxicity of excitatory amino acids, increased ion flow, mitochondrial damage, and the MAPK signaling pathway.

In this study, hippocampal HE staining of the epilepsy + DPCPX group showed that neuronal arrangement was disordered, with cell edema, cytoplasmic vacuoles, degeneration, and other structural damages. TUNEL assay showed that both neuronal apoptosis and Al significantly increased $(p<0.05)$. In the epilepsy + 2-CAdo group, the cell arrangement was basically regular and orderly, and structural damages such as cellular edema and degeneration were alleviated. Furthermore, neuronal apoptosis and Al significantly decreased $(p<0.05)$. Hence, AA1R exerted neuroprotective effects on hippocampal neuronal injury after lithium chloride-pilocarpine-induced epilepsy, being closely related to neuronal apoptosis, which could be antagonized.

Apoptosis is a complex protease cascade reaction mediated by the caspase family, especially caspase- 3 . Herein, compared with the epilepsy group on days 14 and 30 , the caspase- 3 expression levels of the epilepsy + DPCPX group were significantly upregulated $(p<0.05)$, but those of the epilepsy +2 -CAdo group were significantly downregulated $(p<0.05)$. Likewise, Faherty et al. established a rat model of kainic acidinduced epilepsy and found that caspase- 3 activity in 
Figure 3. Effects of A1R on caspase-3 protein expression after injury detected by Western blot. (a) Normal control group, (b) epilepsy group, (c) epilepsy + DPCPX group, (d) epilepsy + 2-CAdo group. *Comparison between normal control and epilepsy groups, $p<0.05$; \#comparison between epilepsy and epilepsy + DPCPX groups, $p<0.05 ; \Delta$ comparison between epilepsy and epilepsy + 2-CAdo groups, $\mathrm{p}<0.05$.

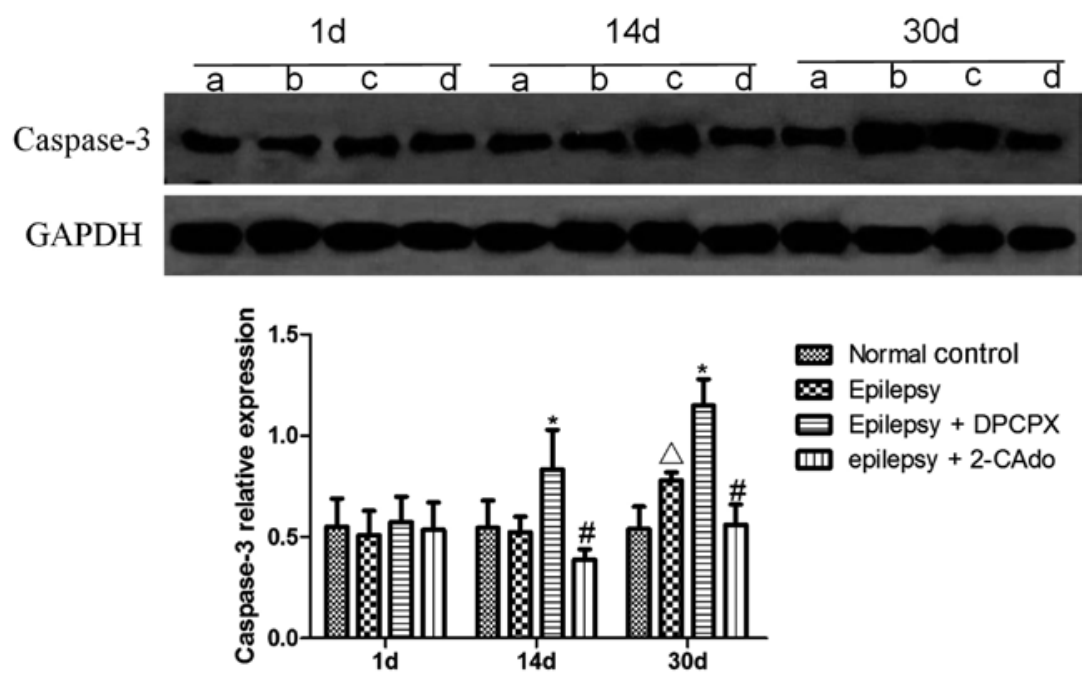

Figure 4. Effects of A1R on GABA receptor expression after injury detected by Western blot. (a) Normal control group, (b) epilepsy group, (c) epilepsy + DPCPX group, (d) epilepsy + 2-CAdo group. *Comparison between normal control and epilepsy groups, $p<0.05$; \#comparison between epilepsy and epilepsy + DPCPX groups, $p<0.05$; $\Delta$ comparison between epilepsy and epilepsy + 2-CAdo groups, $p<0.05$.
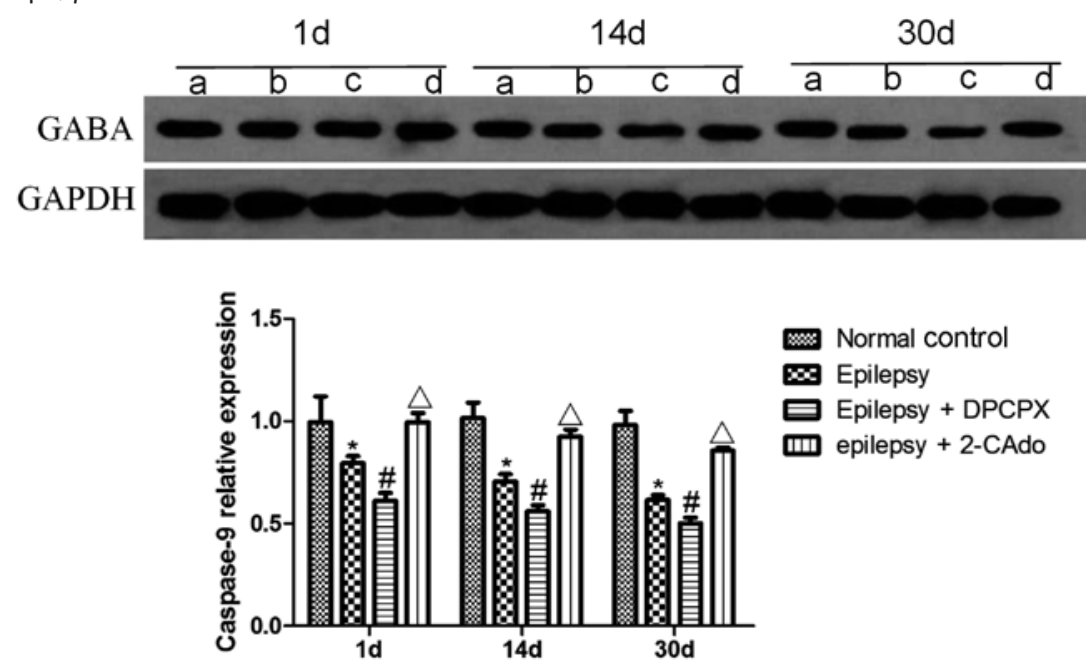

the hippocampal area was significantly enhanced in day 30, suggesting that neuronal apoptosis was closely associated with caspase-3 expression changes. In contrast, AA1R reduced the expression of caspase-322.

As an inhibitory neurotransmitter of the CNS, GABA receptor works mainly through chloride ion channels. In this study, compared with the epilepsy group at the same time point, the GABA expression level of the epilepsy + DPCPX group was significantly downregulated ( $p<0.05$ ), whereas that of the epilepsy +2 -CAdo group was significantly upregulated $(p<0.05)$. Similarly, Reid et al. reported that AA1R elevated chloride ion permeability by regulating GABA level, thus inducing hyperpolarization of intracellular membrane and producing inhibitory postsynaptic potential to combat epilepsy ${ }^{23}$. 
In summary, AA1R had neuroprotective effects on hippocampal neuronal damage after induction of epilepsy by lithium chloride-pilocarpine, mitigating cell edema, and neuronal apoptosis. Nevertheless, this study was limited because we studied the role of $A A 1 R$ in the lithium chloride-pilocarpine-induced epilepsy model only, so other models of epilepsy are needed to unravel the underlying mechanism. The findings provide a novel idea for developing new neuroprotective therapies in clinical practice.

\section{REFERENCES}

1. Heck CN, King-Stephens D, Massey AD, et al. Two-year seizure reduction in adults with medically intractable partial onset epilepsy treated with responsive neurostimulation: final results of the RNS system pivotal trial. Epilepsia. 2014;55: 432-41.

2. Nascimento FP, Macedo-Júnior S], Pamplona FA, et al. Adenosine A1 receptor-dependent antinociception induced by inosine in mice: pharmacological, genetic and biochemical aspects. Mol Neurobiol. 2015;51:1368-78.

3. Dennissen FJ, Anglada-Huguet M, Sydow A, Mandelkow E, Mandelkow EM. Adenosine A1 receptor antagonist rolofylline alleviates axonopathy caused by human tau $\triangle \mathrm{K} 280$. Proc Natl Acad Sci U S A. 2016;113:11597-602.

4. Rivera-Oliver M, Díaz-Ríos M. Using caffeine and other adenosine receptor antagonists and agonists as therapeutic tools against neurodegenerative diseases: a review. Life Sci. 2014; 101:1-9.

5. Hohoff C, Garibotto V, Elmenhorst D, et al. Association of adenosine receptor gene polymorphisms and in vivo adenosine A1 receptor binding in the human brain. Neuropsychopharmacology. 2014;39:2989-99

6. Serpa A, Correia S, Ribeiro JA, Sebastião AM, Cascalheira JF. The combined inhibitory effect of the adenosine A1 and cannabinoid CB1 receptors on CAMP accumulation in the hippocampus is additive and independent of $\mathrm{A} 1$ receptor desensitization. BioMed Res Int. 2015;2015:872684.

7. Thomas TP, Shih TM. The Use of Adenosine Agonists to Treat Nerve Agent-Induced Seizure and Neuropathology. United States: US Army Medical Research Institute of Chemical Defense Aberdeen Proving Ground United States; 2016.
8. Stockwell J, Jakova E, Cayabyab FS. Adenosine A1 and A2A receptors in the brain: current research and their role in neurodegeneration. Molecules. 2017;22:E676.

9. Cheng $P$, Ren $Y$, Bai S, et al. Chronic cerebral ischemia induces downregulation of $\mathrm{A} 1$ adenosine receptors during white matter damage in adult mice. Cell Mol Neurobiol. 2015;35:1149-56.

10. Hu J, Quick MW. Substrate-mediated regulation of gammaaminobutyric acid transporter 1 in rat brain. Neuropharmacology. 2008;54:309-18.

11. Stubbs B, Vancampfort D, Rosenbaum S, et al. An examination of the anxiolytic effects of exercise for people with anxiety and stress-related disorders: a meta-analysis. Psychiatry Res. 2017; 249:102-8.

12. Racine RJ. Modification of seizure activity by electrical stimulation. II. Motor seizure. Electroencephalogr Clin Neurophysiol 1972;32:281-94

13. Zhong M, Song WL, Xu YC, Ye Y, Feng LY. Paeoniflorin ameliorates ischemic neuronal damage in vitro via adenosine $A 1$ receptor-mediated transactivation of epidermal growth factor receptor. Acta Pharmacol Sin. 2015;36:298-310.

14. EuroEPINOMICS-RES Consortium, Epilepsy Phenome/Genome Project, Epi4K Consortium. De novo mutations in synaptic transmission genes including DNM1 cause epileptic encephalopathies. Am J Hum Genet. 2014;95:360-70.

15. Fisher RS, Acevedo C, Arzimanoglou A, et al. ILAE official report: a practical clinical definition of epilepsy. Epilepsia. 2014;55:475-82.

16. Yin D, Liu YY, Wang TX, et al. Paeoniflorin exerts analgesic and hypnotic effects via adenosine A1 receptors in a mouse neuropathic pain model. Psychopharmacology (Berl). 2016;233: 281-93.

17. Liu XH, Xiang SJ, Qi Y. Histopathological changes of hippocampus after acute epilepsy induced by pentylenetetrazole in rats. Chin Pharmacol Bull. 2015;31:514-8.

18. André V, Dubé C, François J, et al. Pathogenesis and pharmacology of epilepsy in the lithium-pilocarpine model. Epilepsia. 2007; 48 Suppl 5:41-7.

19. Hanaya R, Sasa M, Sugata S, et al. Hippocampal cell loss and propagation of abnormal discharges accompanied with the expression of tonic convulsion in the spontaneously epileptic rat. Brain Res. 2010;1328:171-80.

20. Patel HC, Ross FM, Heenan LE, et al. Neurodegenerative actions of interleukin-1 in the rat brain are mediated through increases in seizure activity. J Neurosci Res. 2006;83:385-91.

21. Tan Z, Sankar R, Shin D, et al. Differential induction of p53 in immature and adult rat brain following lithium-pilocarpine status epilepticus. Brain Res. 2002;928:187-93.

22. Faherty CJ, Xanthoudakis S, Smeyne RJ. Caspase-3-dependent neuronal death in the hippocampus following kainic acid treatment. Brain Res Mol Brain Res. 1999:70:159-63.

23. Reid CA, Kim T, Phillips AM, et al. Multiple molecular mechanisms for a single GABAA mutation in epilepsy. Neurology. 2013;80:1003-8. 